\title{
Redes sociales en una investigación de migración indígena: el caso de Manaus
}

\author{
Leonardo H. G. Fígoli* \\ Dimitri Fazito**
}

\begin{abstract}
Este trabajo pretende discutir algunos aspectos de método en el mapeo de redes sociales con migrantes indígenas, a partir de una investigación etnográfica realizada con un grupo de migrantes indios (Alto Río Negro) y residentes en la ciudad de Manaus (Amazonia). El trazado de las redes sociales fue una herramienta crucial en campo, permitiendo identificar los individuos dispersados en el lugar de destino de la migración, obtener datos sobre los modos de desplazamiento o instalación de los migrantes en el medio urbano. Si, por una parte, se mostró un importante instrumento de investigación, se reveló también como noción esencial desde el punto de vista teórico, ya que las propias redes diseñadas constituían una fuerte evidencia de la cohesión social, de la manutención y reelaboración de los lazos étnicos en el contexto urbano. Las redes mapeadas, por ejemplo, revelaron la maleabilidad de las fronteras étnicas diseñadas por los grupos, resultado de las relaciones sociales desarrolladas durante el trayecto migratorio, al paso que nos obligaba a aprehender el fenómeno étnico desde el punto de vista de una genuina antropología relacional. Hoy, las nuevas tecnologías informatizadas permiten la mejor visualización y el análisis más detallado de las redes sociales (ARS), que el que fue posible realizar manualmente en la época de la investigación (1980). Los nuevos recursos hacen aún más importante esa herramienta en la investigación etnográfica. Discutir las múltiples posibilidades y aplicaciones en la investigación de flujos migratorios indígenas es lo que proponemos en este trabajo.
\end{abstract}

Palabras-clave: migración indígena; redes sociales; Amazonia; identidad étnica; urbanización.

\section{Introducción: problemática y metodología}

El trabajo que presentamos es resultado de una revisión, a luz de las nuevas tecnologías informatizadas destinadas al análisis de redes (ARS), de datos obtenidos en una investigación etnográfica realizada en los años 1980 y 1981 (Fígoli, 1982), con inmigrantes indios, oriundos del Valle del Río Negro (Noroeste de Amazonas), asentados en la ciudad de Manaus, Amazonia brasileña. Los datos de las redes sociales recogidos durante la investigación no fueron divulgados completamente en el estudio original, ya que la dificultad de trabajar manualmente con una matriz de datos bastante significativa obligó a utilizar la noción de red en su sentido metafórico y a presentar solamente una descripción general

\footnotetext{
* Doctor en Antropología Social (UnB), Profesor Asociado del Departamento de Sociología y Antropología - Universidad Federal de Minas Gerais (UFMG) - Brasil.

** Doctor en Demografía, Centro de Planificación Regional (Cedeplar) - Universidad Federal de Minas Gerais (UFMG) - Brasil.
} 
de la red indígena urbana, observada con datos cuantitativos globales. ${ }^{1}$ Las nuevas tecnologías informatizadas posibilitaron la revisión y análisis detallado de aquellas informaciones, reforzando con datos más precisos los argumentos defendidos en el estudio original, detectando nuevas características de la red.

El trabajo de campo se realizó en dos etapas. Como a través de la prensa habíamos tomado conocimiento de la existencia de un número expresivo de indios (10.000) de varias etnias, residiendo en la ciudad de Manaus, buscamos en un primer momento identificar los grupos étnicos "destribalizados" -así rotulados por la prensa local- y evaluar el peso demográfico correspondiente. Realizamos esta parte de la investigación en equipo, con otros dos participantes del proyecto, durante los meses de noviembre y diciembre de 1980 . Aunque constatamos, sin demora, que la cifra divulgada carecía de fundamento objetivo, verificamos la presencia de diversos grupos indígenas, provenientes de diferentes sub-regiones amazónicas, siendo los más relevantes: Apurinã, Mundurukú, Mura, Sateré Mawé y un conjunto de "categorías étnicas", agrupadas bajo la denominación regional Indios del Alto Río Negro. En esta etapa, una vez contactados algunos pocos individuos de cada grupo, obtuvimos las primeras informaciones sobre el número aproximado de inmigrantes, así como sobre la antigüedad y las formas de la migración. Después de esta primera relevación se definieron las investigaciones que realizaríamos individualmente.

Durante un breve intervalo en Brasilia, el equipo de trabajo decidió que abordaríamos el fenómeno migratorio por grupo étnico. Aún con pocas, pero promisorias informaciones sobre la existencia de un número significativo de individuos pertenecientes a varias categorías étnicas del Alto Río Negro, decidimos dedicar nuestra investigación al grupo de indios emigrantes de esta región. De regreso a Manaus, la investigación etnográfica se llevó a cabo de enero a abril de 1981.

La primera dificultad que enfrentamos para realizar el trabajo de campo fue la gran dispersión urbana de los migrantes del Río Negro, a diferencia de los otros grupos en estudio muy concentrados en pocas áreas de la ciudad. La gran diseminación espacial obligaba abandonar cualquier intención de obtener un registro total (censo) del contingente poblacional pero, en compensación, creíamos poder localizar, cuantificar y determinar la composición de la población migrante por medio del mapeo sistemático de la red social urbana, o sea, a través del seguimiento de los lazos interpersonales de los migrantes.

La teoría antropológica del contacto interétnico era el horizonte teórico desde el que buscábamos comprender las causas y las características del fenómeno de la migración indígena. Entendíamos este desplazamiento poblacional como un hecho social, mucho más que como un evento de causas económicas ${ }^{2}$, o sea, defendíamos que, ante todo, debía considerarse la migración como un fenómeno social íntimamente relacionado con la situación de contacto interétnico, que caracterizábamos como marcada por relaciones de contradicción, histórica y estructural entre la sociedad indígena y la

\footnotetext{
${ }^{1}$ El estudio formó parte de un proyecto de investigación del Departamento de Ciencias Sociales de la Universidad de Brasilia (UnB), Brasil, coordinado por Roberto Cardoso de Oliveira, Roque de Barros Laraia y Alcida R. Ramos. Patrocinado por el Consejo Nacional de Desarrollo Científico y Tecnológico (CNPq). La investigación abrigó varios proyectos individuales, articulados alrededor de la problemática de las relaciones interétnicas y sus representaciones ideológicas en la Amazonia urbana.

2 Dice Manuel Castells acerca de acelerado crecimiento urbano en Latinoamérica: "La emigración es un acto social y no una consecuencia mecánica de un desequilibrio económico. Su análisis, fundamental para la comprensión de la urbanización, requiere un esfuerzo teórico especial a ese nivel”. Más adelante, refiriéndose a las causas de la migración, critica aquellas posturas que las hacen residir en la supuesta "difusión de los valores urbanos" y agrega: "si en ciertas zonas rurales existe 'difusión urbana', se debe al hecho que las bases estructurales de la nueva situación han desorganizado los sistemas culturales tradicionales" (1972: 118 resalte nuestro). En el caso de la migración indígena la difusión de pautas culturales urbanas está estrechamente vinculada a la forma que tomó el contacto, siendo ésta y no aquella la base sobre la cual deben determinarse las causas del fenómeno migratorio.
} 
sociedad nacional (Cardoso de Oliveira, 1972:30). ${ }^{3}$

Desde esa perspectiva, tratamos de comprender las especificidades de la migración en todas sus fases: desde la salida de los migrantes hasta la inserción final en la ciudad. ${ }^{4} \mathrm{Al}$ considerar el fenómeno migratorio y el grupo indígena desplazado en el contexto de las relaciones interétnicas, asumimos que la misma migración se presentaba como un evento social, también marcado por relaciones de conflicto, del tipo dominación-sujeción. De ese modo, la condición de minoría vivida por el grupo -cuando el origen o las marcas "raciales" del grupo étnico son invocadas por la sociedad políticamente mayoritaria para discriminarlo, segregarlo o perseguirlo, alimentando sobre él una gama amplia de prejuicios- sería, en principio, paradójicamente responsable por la no ocurrencia, en el medio urbano, de la anunciada desintegración o desorganización social, apuntada por el término "destribalización" (Cardoso de Oliveira, 1968:224).

\section{El proceso migratorio: motivación, desplazamiento e integración}

Los indios del noroeste amazónico, conocidos genéricamente como Tukano, viven en pequeños agrupamientos locales, lingüísticamente diferenciados, asentados a lo largo de los ríos Tiquié, Uaupés, Içana afluentes del Río Negro. Los grupos toman la forma de categorías étnicas con identidades propias, se ordenan jerárquicamente, comparten algunas tradiciones y mantienen intercambios exogámicos.

La literatura etnográfica ha sido muy obscura en lo que respecta a la clasificación étnica de los grupos indígenas del Alto Río Negro. Hay interpretaciones divergentes acerca de las vinculaciones que mantienen esas unidades sociales que componen la totalidad indígena del valle del Río Negro, siendo generalmente explicaciones parciales o mutuamente exclusivas. ${ }^{5}$ Según Hugh-Jones (1979: 22), hay dos grandes tendencias de explicación: por una parte, autores que acentúan el aspecto de la unidad y similitud de aquellos grupos indígenas y, por otra, los que destacan el carácter diverso y específico de cada unidad social.

Conceptos como tribu, clanes, fratrías, grupos exogámicos, agregados lingüísticos, han sido utilizados para describir los límites y articulaciones de los grupos. Enfatizando o no el aspecto diferenciador del lenguaje -el término de grupo exogámico- sirvió de base a la mayor parte de los investigadores para establecer las frontera grupales y explicar los niveles de inclusividad internos (sibs jerárquicas). La búsqueda de datos objetivos (lingüísticos, culturales u organizacionales) que refuercen las señales diacríticas sociales (las identificaciones grupales) se ha mostrado insuficiente y, la mayoría de las veces, como

\footnotetext{
${ }^{3}$ Sobre la migración indígena de los indios del Río Negro, Gabriele Brandhuber (1999) hizo observaciones posteriores a las nuestras, en São Gabriel da Cachoeira-AM, entre 1996 y 1997. La autora, como nosotros, también considera insuficiente el factor económico para explicar el fenómeno pero señala, como posibles causas, la tradicional movilidad espacial de estos grupos bien como sus frecuentes conflictos y fenómenos de fisión, combinados con deseos (recientes) de ascensión social. Aunque no disentimos esencialmente de sus argumentos, nos parece, en cambio, que debe considerarse, ante todo, la situación de contacto interétnico una vez que esa contradicción es total y "totalizadora", afectando con gran poder todas las dimensiones de la vida indígena.

${ }^{4}$ Contábamos con escasos antecedentes de estudios de población indígena urbana en el Brasil que sirviesen de marco referencial, con excepción de la investigación realizada por Roberto Cardoso de Oliveira con los Terêna (1968) que trataba, principalmente, del proceso de urbanización e integración de migrantes en la sociedad de clases. Ese estudio revelaba formas de persistencia de lazos tribales en medio a un proceso de alto nivel de aculturación, en una situación urbana, sin que ocurriera pérdida de identificación étnica de sus miembros.

5 De ese modo, se llegó a una gran variedad de formas clasificatorias basadas en criterios lingüísticos, como los estudios de Sorensen (1967) o Jackson (1974); en trazos culturales y organizacionales, como los trabajos de Goldman (1963), S.Hugh-Jones (1979), Reichel-Dolmatoff (1973), Galvão (1979), para citar los más importantes. A pesar de los numerosos esfuerzos por delimitar las "fronteras" grupales, las clasificaciones sugeridas se han deparado con innumerables problemas de inadecuación de tales criterios con la realidad estudiada. Sea enfatizando los límites grupales por barreras lingüísticas ("agregados lingüísticos"), sea distinguiéndolos por trazos de organización social según las reglas de exogamia y patrilinealidad, se ha llegado a una abundante terminología diversa y divergente para dar cuenta de la complejidad de relaciones que envuelven a las referidas unidades sociales de la región norte del Río Negro.
} 
una dificultad para la comprensión de las representaciones mediadoras de las relaciones intergrupales.

Consideramos el proceso migratorio como un acto social compuesto de tres fases (Eisenstad, 1954:1): a) motivación para migrar: comprende las disposiciones o necesidades que pueden haber inducido a la población indígena a abandonar sus lugares de origen para dirigirse a los centros poblados amazónicos; b) estructura del proceso migratorio: considera las características de la población migrante, sus lugares de partida, las modalidades, rutas y etapas migratorias; c) integración de los migrantes a la estructura organizacional urbana a través de indicadores como forma de residencia y de trabajo.

Sobre el primer aspecto de la migración, sabíamos que no era un fenómeno reciente, pues existen noticias de grandes movimientos de población indígena del Alto Río Negro desde los primeros momentos del contacto. ${ }^{6}$ Cada uno de los ciclos económicos por los cuales atravesó la región amazónica, produjo, invariablemente, fuertes desplazamientos de indios a consecuencia de la violenta penetración de frentes nacionales en sus territorios. En el último siglo, el frente misionero representó el segmento más importante, casi único, de la sociedad nacional presente en los territorios indígenas del aislado noroeste amazónico. La migración es un fenómeno recurrente, hecho históricamente relacionado con el contacto entre indios y blancos, indiscernible de la violenta situación de dominación a que vienen siendo sometidos.

En el último siglo, las misiones religiosas forzaron la reunión de individuos de diferentes categorías étnicas a convivir en los espacios restrictos de las misiones religiosas, provocando una situación de intenso contacto, directo y cotidiano, antes inexistente, que exigió la adopción de nuevas conductas interpersonales. Por una especie de migración forzada hacia las misiones, los individuos transplantados pasaron a formar parte de un grupo heterogéneo, artificialmente uniformizado por la acción de los religiosos bajo el lema "en la misión todo el mundo es igual". Sin duda, los centros misioneros tendieron así el primer puente entre el mundo tribal y el mundo "civilizado". Es allí donde los jóvenes pasan a familiarizarse con el "mundo moderno" y urbano, a través de los bienes de consumo, la presencia de autoridades civiles y militares, de la comunicación regular con las ciudades, de la posibilidad de empleo asalariado o aún en la "animación" deportiva o recreativa promovida por las misiones.

El proceso de acelerada aculturación, producto de una rígida instrucción escolar, más la convivencia con la situación casi urbana de las misiones, genera una clara conciencia de la oposición existente entre el mundo indígena y el mundo de los blancos. Así, el simple desplazamiento de la aldea para la misión resulta en una primera y fundamental experiencia migratoria para los indios. Se trata de una aproximación y exposición a la vida urbana, sin salir de los contornos de la propia área, que facilita la disminución de la "distancia estructural" entre el mundo tribal y el urbano, tornándose factor de estímulo, sino expulsor, del proceso migratorio para la ciudad.

Aunque las motivaciones para emigrar que nos fueron relatadas no diferirían de muchas otras corrientes de migración (motivos económicos, sociales o políticos), sin embargo, suponen ciertas representaciones comunes íntimamente asociadas a la situación de contacto. Si la necesidad de emigrar es muchas veces producto de las escasas opciones que ofrece el sistema de relaciones que predomina entre indios y blancos en la región, estas están asociadas por los indios a los misioneros religiosos, que se les figuran como únicos administradores de esas oportunidades. Desde la perspectiva de los agentes, la migración es vista como un hecho que resulta menos de la atracción que ejerce la ciudad y más como alternativa

\footnotetext{
${ }^{6}$ Se tiene noticias de movimientos de población indígena en la región desde los famosos desplazamientos forzados por los blancos para reclutar mano de obra indígena llamados "descimentos", hasta los grandes movimientos milenaristas conocidos como de los "falsos cristos".
} 
a la actual situación de dominación étnica, marcada por condiciones de existencia vistas como inherentes al contacto. En los casos de conflicto abierto con los misioneros, la emigración es aún vivida como un acto necesario, producto de la sujeción a la "autoridad misionera". En suma, las explicaciones que los agentes dan sobre la génesis de la migración, revelan un modo perceptivo común, una lógica étnica, por el cual es vista como un hecho indiscernible de la situación de contacto que los somete. Estas representaciones proveen a los agentes una coherencia relativa sobre el propio fenómeno migratorio, visto como el producto más inmediato de las relaciones de dominación existentes de la sociedad nacional sobre la sociedad indígena.

El número de indios oriundos de la región del Alto Río Negro y residentes en Manaus que obtuvimos a través del mapa de las redes, no representa la totalidad de los migrantes. Aún así, pudimos identificar un grupo bastante significativo, la gran mayoría proveniente del llamado triángulo misionero ${ }^{7}$, fuertemente vinculados por una extensa red social urbana, de lazos múltiples y singularizada por su carácter étnico. ${ }^{8}$ Como no se realizó un censo, el número de individuos con adscripciones étnicas propias del Río Negro y residentes en Manaus en el momento de la investigación, se obtuvo a través de los lazos sociales mantenidos por los migrantes, lo cual mostró la existencia de una red extensa con gran conectividad, pues a través de ella se relacionaban 11 categorías étnicas diferentes, lo que también confirmaba la persistencia de los vínculos establecidos en las aldeas de origen, así como los producidos durante la migración, sea durante la estadía en las misiones religiosas, sea los trabados en los puntos de paradas o escalas, sea en la misma ciudad de destino.
Del punto de vista del volumen y la composición por sexo y edad de la corriente migratoria, la gradual saturación de la red tribal, alcanzada hacia el final del trabajo de campo, permitió concluir que habíamos alcanzado valores próximos del total de residentes de aquella región, así pudimos establecer que el fenómeno afectaba por igual a ambos sexos. Cuando consideramos las clases de edad, $81 \%$ de los instalados en la ciudad tenían entre 20 y 39 años de edad, distribuidos proporcionalmente entre ambos sexos. Podíamos concluir que la migración era maciza, compuesta de individuos jóvenes y de ambos sexos. El número pequeño de migrantes antiguos encontrados permitía inferir que el flujo se había intensificado en los años precedentes a la investigación.

El análisis del proceso migratorio permitió abordar detalladamente las características de la población que se radica en la ciudad, así como las formas de traslado prevaleciente entre diferentes generaciones de migrantes. Los datos cualitativos obtenidos por medio de historias migratorias y laborales revelaron que los antiguos migrantes lo hicieron de manera independiente y por etapas; los más recientes se desplazaron directamente a Manaus con participación de agencias interculturales. En todos los casos, sin embargo, la solidariedad grupal del movimiento migratorio se manifestó en la intensa activación de las redes sociales tribales de lazos diversos. Observamos también que la creciente participación de agencias nacionales intervenía como orientadora del flujo migratorio, imponiendo formas a la movilidad de los grupos. Consecuentemente, la migración se presentaba como resultante de un estado de conflicto étnico, pero al mismo tiempo lugar de reproducción de los mecanismos

\footnotetext{
7 Las misiones religiosas a que aludimos son tres: Yauareté, Taraquá y Parí-Cachoeira, sobre los ríos Uaupés y Tiquié, afluentes del Río Negro.

8 Nos apoyamos en el concepto de red utilizado por Barnes (1969) y elaborado anteriormente por Bott (1957). Ambos conciben esta red como una serie de relaciones que un individuo configura alrededor suyo. Por otra parte, Mayer y Epstein, mostraron cómo determinados tipos de migrantes se "encapsulan" en una hermética red de relaciones personales y cómo esta red, que se prolonga para la zona de origen sirve para proteger sus miembros y para potenciar sus orientaciones originales (Mitchell, J.C., 1980:71).
} 
de dominación, de los cuales el migrante buscaba escapar. Desde el punto de vista de los actores, la migración ocurre en un espacio étnicamente dividido, en un espacio regulado por el blanco y apenas atravesado por las redes sociales indígenas.

Por último, utilizamos como indicadores las formas de residencia y de trabajo para evaluar el grado de incorporación al sistema organizacional urbano. Si bien la incorporación de los migrantes indígenas a la estructura de organización urbana es típica de lo que conocemos como "urbanización dependiente", se pudo obtener de las historias de vida y de trabajo, mecanismos específicos de inserción, pues, a diferencia de las estratos urbanos llamados "marginales", la fijación en la ciudad y la obtención de empleo entre los indios requiere una malla de relaciones sociales intra e interétnica que le otorga una especificidad étnica a dicho proceso, distanciándolos de otras fracciones sociales en condiciones homólogas en la estructura social. Se ve aflorar también una óptica étnica, a través de la cual se vive la adecuación-inadecuación al mundo de los blancos, perspectiva que expresa no sólo la idéntica posición en la estructura social, en los estratos más bajos de la sociedad de clases, sino que contiene el sentido de una idéntica trayectoria colectiva.

\section{Consideraciones metodológicas: redes sociales totales y egocentradas}

Cuando se emprendió la investigación, en 1980, la metodología del Análisis de Redes Sociales (ARS) aún no se había consolidado y se carecía de equipamientos y programas computacionales capaces de procesar ese tipo de informaciones. Las nuevas tecnologías informatizadas, así como los avances de la estadística de redes, posibilitaron hacer una revisión de los datos originales y un análisis detallado de las informaciones recogidas sobre los inmigrantes a Manaus, utilizando nociones e instrumentos de medición y visualización más precisos. A partir de la experiencia de esta investigación, de los datos revistos a la luz de las nuevas tecnologías, queremos discutir la posibilidad de la aplicación de las técnicas de redes sociales en los estudios demográficos con poblaciones indígenas, especialmente para acompañar desplazamientos migratorios. En otras palabras, con este trabajo queremos evaluar la compatibilidad del Análisis de Redes Sociales en el terreno de los estudios demográficos en la investigación antropológica, particularmente con sociedades indígenas.

En primer lugar, es necesario subrayar que el Análisis de Redes Sociales opera desde una perspectiva esencialmente relacional, o sea, se trata de un análisis que centra toda la atención en los lazos o conexiones existentes entre los actores sociales, sean estos individuos o grupos, considerados, así, como nodos o vértices de las redes que las relaciones constituyen. Por otro lado, es también un análisis estructural, ya que busca identificar los patrones de relaciones, es decir, las invariantes o regularidades emergentes en el contexto de la interacción investigada, que configuran las topologías de redes (estructuras invariantes que suelen ser clasificadas y mensuradas con técnicas apropiadas). Así, la diferencia elemental entre el ARS y el análisis estadístico tradicional resulta en la consideración de relaciones necesariamente asumidas como "eventos interdependientes", asociadas a los atributos categóricos.

En segundo lugar, es importante señalar que existen dos estrategias de investigación, las cuales constituyen el cerne del ARS: por una parte, la perspectiva del análisis de las redes totales; por otra parte, el análisis de las redes egocentradas (Hanneman, 2001; Knoke y Kuklinsky, 1983; Wasserman \& Faust, 1994; Scott, 2000). En la perspectiva de las redes totales, se empieza el análisis estructural, partiendo de la colección amplia de los actores (nodos) y las relaciones (lazos) específicas que los vinculan, las cuales serán responsables de la definición de las fronteras de la red social a la que llega el investigador. Así, el analista podrá comparar las posiciones estructurales diferenciadas de los actores, según las medidas de la red total, y estudiar los efectos estructurales de los patrones de relación sobre los comportamientos individuales 
de los actores. Esta perspectiva también es conocida como top-down (Hanneman, 2001), pues permite al analista controlar las variables de composición de la red (atributos) por las variables estructurales de la topología reticular, y evaluar las asociaciones y causalidad.

En la segunda perspectiva, o sea, la de las redes egocentradas, el analista empieza la definición de la red por los actores individuales y sus relaciones personales inmediatas; así, los lazos recogidos son exclusivamente aquellos indicados por los actores. La suma de redes egocentradas generalmente se limita a las informaciones de Ego y pueden no permitir un análisis estructural completo (Wellman, 2002; Scott, 2000:30-2). Actualmente, algunas técnicas para el análisis de redes personales estructurales (McCarty et al. 1997; McCarty, 2002) permiten una investigación segura de los efectos estructurales sobre los comportamientos a partir de redes egocentradas (esta alternativa es conocida como el análisis de redes personales, diferente del análisis clásico de egocentradas). En este trabajo presentamos un análisis compuesto por la perspectiva de redes totales y complementado por redes egocentradas, pues las informaciones recogidas originalmente fueron reconstituidas y adaptadas a la perspectiva de actores indígenas centrales.

Un gran problema lo constituye el muestreo de los inmigrantes y, por tanto, las limitaciones del ARS para generalizaciones. Con frecuencia, el muestreo en ARS hace uso de la técnica de snowball para determinación de las fronteras de la red social. De acuerdo con Billsborrow (1997), la técnica de snowball es más apropiada para la investigación de poblaciones raras u ocultas, como es el caso de poblaciones migrantes. El límite, entretanto, es la generalización, pues las conclusiones son específicas del universo investigado, o sea, equivale a las fronteras del estudio de caso. Sin embargo, mientras el estudio de caso sea representativo de un contexto social e histórico, el análisis se muestra efectivo y relevante.

Así, las informaciones recogidas por Fígoli representan una red total de inmigrantes indígenas en Manaus en los años 1980-1 (la red total no implica la totalidad de inmigrantes, no necesita ser un censo). Además, el método empleado para recoger las informaciones posibilitaron también la reconstitución de redes egocentradas de algunos actores indios, en general, los informantes-llave (aunque esas egocentradas, es necesario aclarar, no pueden ser consideradas redes personales completas, como las desarrolladas recientemente por McCarty, 2002).

Las posibilidades teóricas, metodológicas y conceptuales del ARS para la demografía son variadas y poco conocidas. Podemos decir que para el estudio más apropiado y denso de las poblaciones pequeñas y raras, como los indígenas e inmigrantes, la técnica de snowball combinada con el análisis de las redes egocentradas (especialmente las redes personales de McCarty) y totales son muy interesantes para la evaluación de las correlaciones y asociaciones causales entre variables categóricas y estructurales, e incluso las posibilidades de estimativas de poblaciones raras con técnicas especiales del ARS (Killworth et al., 1998).

Todavía hay mucho que avanzar en estos abordajes, y esta evaluación de la técnica de redes, empleada a la luz de los nuevos recursos técnicos y de los avances teóricos y metodológicos experimentados por el ARS, es un primer esfuerzo en ese sentido. Para más adelante, se puede imaginar la obtención de nuevos datos de la población indígena migrante del Alto Río Negro, desde la perspectiva de las redes personales, por ejemplo, para sopesar la compatibilidad entre variables de composición y las estructurales. Esta alternativa de investigación podría revelar otros padrones estructurales, propios de estas poblaciones indígenas, y auxiliar al análisis de los efectos demográficos de la migración sobre la organización tribal y la dinámica urbana.

\section{La red tribal urbana mapeada}

De acuerdo con el concepto antropológico de red social (Barnes, 1969), partimos del principio de que los lazos interpersonales de un miembro del grupo formarían parte 
de un sistema total de interrelaciones. Así, esperábamos que el mapeo extenso de nodos y lazos revelase el número aproximado de inmigrantes, pero también informase sobre los límites (fronteras) y articulaciones entre las diversas categorías étnicas, así como sobre la estructura interna de los grupos. De hecho, abordamos el contingente de inmigrantes con esa estrategia, lo cual nos auxilió, primeramente, en la localización de los individuos y los grupos domésticos oriundos del Río Negro, dispersos en 19 barrios diferentes de la ciudad. Por medio de esta técnica de investigación, conseguíamos localizar los individuos y evitar también el escamoteo de la identidad étnica de los entrevistados, manipulación que sabíamos era muy frecuente a consecuencia de la fuerte discriminación impuesta por la sociedad regional a las poblaciones indígenas. Pero, además, la confección de la propia malla se mostraba esencial del punto de vista de la problemática teórica, pues permitiría aprehender empíricamente las fronteras étnicas de los varios grupos, operando fuera del territorio indígena, ya que la sola existencia de la red urbana sería una evidencia del conocimiento y reconocimiento mutuo de los actores, en tanto miembros de las categorías étnicas que integran el grupo Alto Río Negro. Por lo tanto, haciendo el mapa de la red tribal en la ciudad, no solamente conseguíamos paulatinamente localizar y cuantificar los inmigrantes por un medio confiable, sino que también obteníamos simultáneamente una importante evidencia del grado de cohesión social mantenido por el grupo indígena en el contexto urbano.

El diseño y el análisis de la red social urbana del grupo tribal permitieron establecer los vínculos efectivos entre los agentes, y con ello pudimos evaluar la vigencia de las lealtades tribales en las condiciones, generalmente adversas, de la nueva situación urbana. El registro de los lazos interpersonales mostró rápidamente no sólo la alimentación de los vínculos originales de parentesco y amistad, sino también la existencia y mantenimiento de nuevas relaciones trabadas en condiciones generadas por la situación de contacto, como las propiciadas por las misiones, por ejemplo, pero también las ocurridas durante el mismo proceso de desplazamiento migratorio.

El diseño de la trama tribal que obtuvimos, siguiendo las cadenas orgánicas de la red social, evidenció la manutención de una red bastante compleja de lazos múltiples entre los migrantes, establecidos antes y durante el proceso migratorio. En la misma medida que íbamos diseñando la red, orientados por los propios migrantes, obteníamos evidencias para colocar en tela de juicio la idea, bastante común, entre especialistas o no, de que la "destribalización", o sea, el proceso de abandono de las aldeas, sería sinónimo de pérdida de las identidades étnicas.

La red total de los migrantes del Alto Río Negro, instalados en Manaus en la época de la investigación, estaba constituida de 222 individuos, de ambos sexos y provenientes de sub-regiones diversas, pertenecientes a 11 categorías étnicas. El contingente de migrantes instalado en la ciudad de Manaus estaba compuesto por individuos de los siguientes grupos: Tukano, Desano, Tuyuka, Arapaço, Wanano, Pirá-Tapuya, Mirití-Tapuya, Baraçana y Karapaná -de la lengua Tukano-Betoya; Tariano y Baniwa, de la lengua Aruak.

\section{Red total}

Analizaremos ahora, en un primer momento, algunas características estructurales de la red total diseñada y, más adelante, separaremos las redes egocentradas de los principales informantes. El análisis de la red total $(n=222)$ sugiere muy baja densidad global (0.019). Con apariencia de un "queso suizo", la red de los migrantes instalados en Manaus presenta grandes agujeros y pocas acumulaciones localizadas alrededor de unas figuras centrales pero, como veremos adelante, no significa mal conectada, dado el alto grado de cohesión de los actores, o sea, pocos actores conectan muchos individuos (ver Figura 1). En esta situación el rol de los actores intermediarios adquiere importancia fundamental para la disposición de los lazos 
y la estabilidad de los flujos de información. En general, la malla producida por la migración presenta posiciones estructurales intermediarias ocupadas por agentes singulares que actúan cómo brokers e influyen en los flujos y volúmenes de información (Fazito, 2005: 177-8). En este caso, se puede ver que muchos lazos convergen hacia pocos agentes centrales, responsables por la conectividad y cohesión entre los demás actores de la red social.

La figura arriba presenta un dígrafo, o sea, un grafo con lazos diseccionados, de la red total de los indígenas. Este grafo resulta de un análisis multidimensional (MDS), basado en la similitud de la posición estructural entre los actores, o sea, la similitud de la distribución de los actores y sus conexiones en toda la red. Aquí, fue utilizado el algoritmo spring-embedding, de Kamada-Kawai, basado en el análisis multidimensional para dibujar la red (con el software Pajek 1.1), de acuerdo con la proximidad de los actores respecto a sus padrones relacionales. Así, encontramos en al centro del grafo los actores medulares, o sea, aquellos indígenas que sostienen más y diversificadas conexiones con otros actores. Estos actores (en especial 10, 73 y 83) constituyen un grupo de personas muy conectadas entre sí que, además, conectan indirectamente muchos otros actores - generalmente, actores de grupos familiares y/o étnicos específicos. De ese modo, el grafo (Fig.1) sugiere un modelo tipo centro-periferia, donde los actores más conectados están situados en el centro; más lejos, alrededor de ellos, se encuentran los actores menos conectados. Podemos observar diferencias estructurales importantes en los actores más conectados de la periferia, como en los casos 117, 96 y 112, por ejemplo. Estos individuos, aunque sostienen también múltiples conexiones, son menos importantes estructuralmente cuando son comparados a 10, 73 y 83, porque sus conexiones se dirigen hacia actores poco conectados y relativamente aislados en la periferia de la malla social. Por fin, el grafo revela aún la existencia de 5 subcomponentes: la díada 62-210, y los puntos aislados 85, 150 y 163 constituyen subcomponentes periféricos; el gran subcomponente restante, con 217 actores, presenta una conectividad interna fuerte y significativa. Estas diferencias de distribución de los actores y sus conexiones explican por qué la red tiene baja densidad, pero relativa conectividad interna (en especial cuando consideramos el gran subcomponente). Es importante recordar que la densidad indica la existencia de lazos efectivos, comparada

FIGURA 1

Red migratoria total, indios del Alto Río Negro

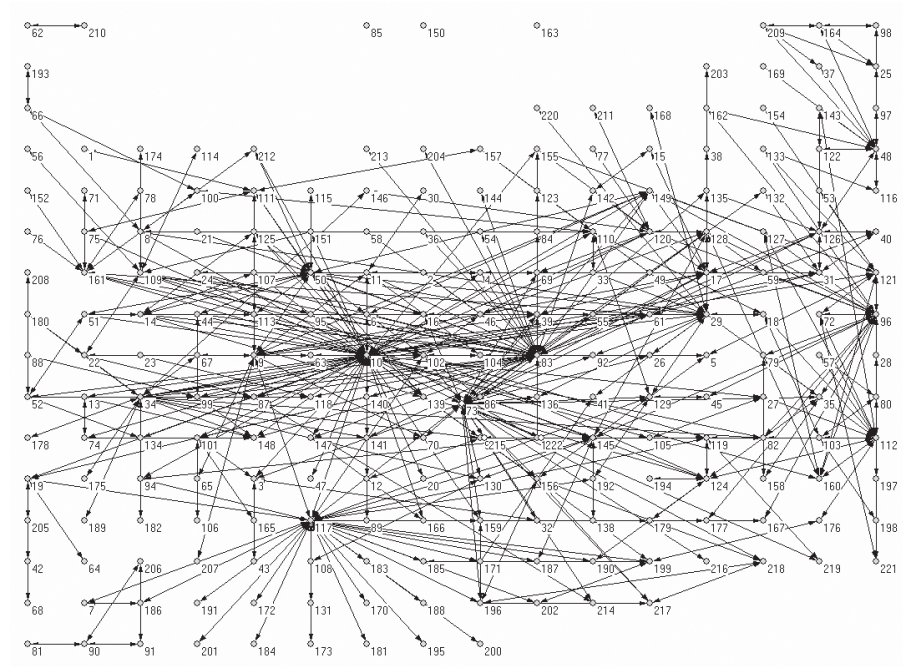


con la proporción de lazos posibles. De ese modo, la densidad es una medida que varía en función del tamaño total de las redes, de manera que cuanto más grandes son (la red en análisis puede considerarse una red de tamaño medio), menores serán las densidades correspondientes (Scott, 2000). Otras medidas reticulares serán necesarias para una evaluación más clara de los padrones estructurales.

La cohesión basada en las distancias geodésicas es relativamente débil (0.311), y el promedio de la distancia entre los agentes de la red es 3.2 pasos. Ambas medidas refuerzan la idea de una red dispersa con pequeños, pero fuertes aglomerados de individuos. Nos parece observar en estas características de la red el hecho de que constituye una trama de relaciones, que teniendo por referencia las relaciones de parentesco originales, tiende a reemplazar los lazos ausentes de consaguinidad o alianza por otras relaciones basadas en la camaradería, la cooperación, la proximidad urbana, etc. Debemos tener en cuenta que los inmigrantes se encontraban diseminados en 19 barrios diferentes, lo cual puede explicar, en parte, la forma que toma la red.

Por otra parte, el análisis de los componentes de la red señala la ocurrencia de 139 cliques (mínimo de 3 agentes) y gran superposición de las tríadas, factor que sugiere una buena conectividad entre los agentes de la red. De hecho, el concepto de "clique" en ARS identifica un sub-grafo máximo, o sea, un subgrupo de actores que tienen necesariamente lazos directos entre sí. Los actores que participan en un clique pueden estar presentes en otros diversos, y así el número de cliques y superposiciones (actores compartidos entre diferentes cliques) indican, por un lado, la fuerza cohesiva de la red total, y de otro lado, la conectividad de los actores y su capacidad de conectar a los otros.

Los índices de red total utilizados también señalan una red poco centrada, o sea, pocos individuos son capaces de alcanzar, con baja intermediación, la totalidad de los nodos. Los índices de centralidad componen una familia de medidas correlacionadas, porque tienen el mismo principio, o sea, buscan revelar la jerarquía estructural de los actores y sus lazos, de los más a los menos conectados en la red. Todos los algoritmos de centralidad (degree, closeness, betweenness, bonacich eigenvector, flow betweenness) buscan determinar una escala entre los nodos, pero también identifican medidas globales de centralización total (Wasserman \& Faust, 1994). Por ejemplo, el índice general de centralización de Freeman (degree) es una medida global de la red y sugiere el grado de concentración de los nodos alrededor de un nodo de posición más central. Las medidas de centralización, como el degree, se basan en el modelo matemático del grafo-estrella, en el cual un nodo central se conecta directamente a todos los demás nodos, pero estos mantienen apenas un lazo directo con el nodo central. Los índices de centralización varían de $0 \%$ a $100 \%$, de modo que el grafoestrella, por ejemplo, constituye la variación máxima de $100 \%$, pues este contiene un sólo nodo, totalmente conectado con los otros, los cuales, a su vez, se conectan únicamente con él. Considerando la red indígena en análisis, la centralización de Freeman revela un índice $28 \%$, sugiriendo una red bastante homogénea, donde todos los nodos tienen una distribución de lazos estructuralmente equivalentes. En ese caso, podemos observar que la mayor parte de los agentes tienen pocos contactos (un promedio de 8 contactos directos con otros nodos), y pocos actores revelan más vínculos que el promedio. Por otro lado, la centralidad presentada por el índice de intermediación (betweenness) es de 38\% y a ella se aplica la misma interpretación. Pero un índice de betweenness más allá de $20 \%$ es considerado elevado, o sea, el índice sugiere que la red como un todo presenta gran potencial de mediación entre sus miembros, de modo que algunos nodos específicos detentan ventajas estructurales para conectar actores que no mantienen lazos entre sí. La ventaja estructural del broker no siempre es percibida por los actores, de manera que muchos individuos poseen esa ventaja estructural, pero no lo saben. 
El análisis de la inserción local de los agentes se concentró en aquellos nodos estructuralmente más importantes, determinada por los índices de centralidad (degree, closeness, betweenness y eigenvector) medidos por el algoritmo "centrality group" del software UCINET6.1 - ese algoritmo mide todos los índices de centralidad normalizados y sugiere una jerarquía estructural de los nodos distribuidos entre la mayor y la menor centralidad detectada en la red total. Estos nodos, distribuidos en orden de centralidad, son B.T. (10), H.H. (83), G.X. (73), M.N. (117), C.D. (34), F.T. (50) y K.M. (96). Después, evaluamos la fuerza de los intermediarios (brokers) con el programa Keyplayer 1.0, que aplica un algoritmo basado en la distribución de las centralidades individuales ponderadas por la centralización global, y reveló cuales son los actores más bien posicionados estructuralmente para intermediación: B.T. (10); H.H. (83); G.X. (73); C.D. (34); M.N. (117); F.T. (50).

Visto que los actores B.T. (10) y H.H. (83) son informantes llaves que ocupan una posición destacada en la jerarquía estructural de las posiciones en la red indígena en Manaus, y además son los actores privilegiados en la intermediación en toda la red, es necesario concentrar el análisis de la perspectiva egocentrada en esos nodos.

\section{Redes egocentradas}

Tanto el análisis de las medidas de red como la representación visual de las redes egocentradas sugieren que los agentes más importantes y preponderantes en la red migratoria son B.T. (10) y H.H. (83). Ambos tienen gran importancia para las conexiones globales de la red migratoria en Manaus, o sea, la díada mantiene relaciones que pueden ser caracterizadas como múltiples con un grado importante de extensión, lo cual les asegura una base adecuada de relaciones indefinidas, necesarias para sustentar el liderazgo que ejercen. Por ejemplo, B.T. (10) está presente en 69 cliques; H.H. (83) está presente en 41 ; y la díada B.T. (10)-H.H. (83) está presente en 25 cliques, o sea $18 \%$ de todos los cliques.

De hecho, el gráfico presenta una instantánea de un momento crítico vivido por los grupos indígenas del Alto Río Negro, generado por una acusación internacional de etnocidio que recaía sobre la congregación religiosa de los misioneros, presentada ante IV Tribunal Russell (Holanda, 1980) por B.T. (10). Sin duda, los lazos del gráfico reflejan un momento de multiplicación de vínculos de B.T (10) a consecuencia del conflicto abierto con el frente religioso, vínculos movilizados por la acción política que vinieron a sumarse a los vínculos constantes de parentesco, lealtades étnicas, etc. (Mitchell, 1969: 26), acción política secundada en el medio urbano por H.H. (83), oriundo de la misma región y aliado estratégico de B.T. en la crítica política y las reivindicaciones indígenas. La mayor parte de los individuos incluidos en la red principal, controlada tanto por B.T. (10) como por H.H. (83), es pasible de ser movilizada ante situaciones de crisis. Es importante aclarar que todo un segmento de la red total mantenía vínculos frecuentes y de carácter múltiple, en cuanto el otro, presentaba relaciones esporádicas. B.T. (10) controlaba una red personal compuesta por 65 actores distribuidos equilibradamente según el sexo, la etnia y la región del origen (Figura 2).

Si analizamos las cualidades de los vínculos entre ego y las personas con quien actuó, el mayor número corresponde a individuos de la misma región de origen, o sea, de la misma aldea o centro misionero. Por lo tanto, la red se presenta como una extensión de la red de parentesco, trazo característico de las redes en sociedades tribales (Barnes, 1969).

En el sociograma egocentrado abajo (Fig.2) se observa que las distribuciones de los contactos personales de B.T. (10) son equilibradas y amplias. Este actor controla una red diversificada de contactos separados según todas las categorías de diversos tipos.

B.T. es el actor aislado en la base del grafo (cuadrado rojo). En el sentido horario se puede ver los actores agrupados según la región de origen migratorio. Así, después 
de B.T. vemos agrupados los miembros de una misma región de origen: el Grupo 1 (São Gabriel da Cachoeira); después, el actor aislado (cuadrado azul), es el único de la red egocentrada de B.T., y constituye el Grupo 2, procedente de la región de Cucuí; o próximo aglomerado de actores es el Grupo 3, procedentes de Taraquá; a seguir la díada aislada forma el Grupo 4, de Maracajá-São Francisco; el Grupo 5 de actores siguientes componen aquellos sin identificación de procedencia; el cuadrado azul aislado en la esquina derecha de la figura constituye el Grupo 6, de la región de Camarus; el gran aglomerado a seguir, es el Grupo 7, procedentes de Parí-Cachoeira; y finalmente, el Grupo 8, de los tres actores en el extremo derecho son procedentes de la región de Yauareté.

Verificamos allí que B.T. mantiene relaciones con indígenas oriundos de diferentes lugares de la región del Alto Río Negro. Más importante aún, se puede ver que en algunos casos (como con la díada Maracajá-Sao Francisco, y los tres actores de Yauareté del último grupo) B.T. funciona como intermediario, una vez que algunos individuos provenientes de una misma región, cuando llegan a Manaus no se conectan directamente. Finalmente, vemos que independientemente de B.T., los indígenas de la región de São Gabriel (Grupo 1), mantienen una interacción muy fuerte, en Manaus, con los migrantes provenientes de Parí-Cachoeira (Grupo 7). Además, los indígenas de São Gabriel, una vez en Manaus, operan como referencia en la red general de contactos indígenas, pues puede verse claramente que todos los grupos mantienen vínculos directos con los diversos actores procedentes de aquella región, y el mismo patrón de intensidad no se observa entre los demás grupos (no se observan tantos lazos entre los otros grupos, independientes del Grupo 1). Como este sociograma egocentrado fue extraído de la rede total, de hecho, muestra la mayor participación de los indígenas de São Gabriel, ya que constituye un importante punto intermedio en el desplazamiento migratorio, para la conectividad de la red en Manaus.

FIGURA 2

Red egocentrada de B.T. (10) según sexo, etnia y región

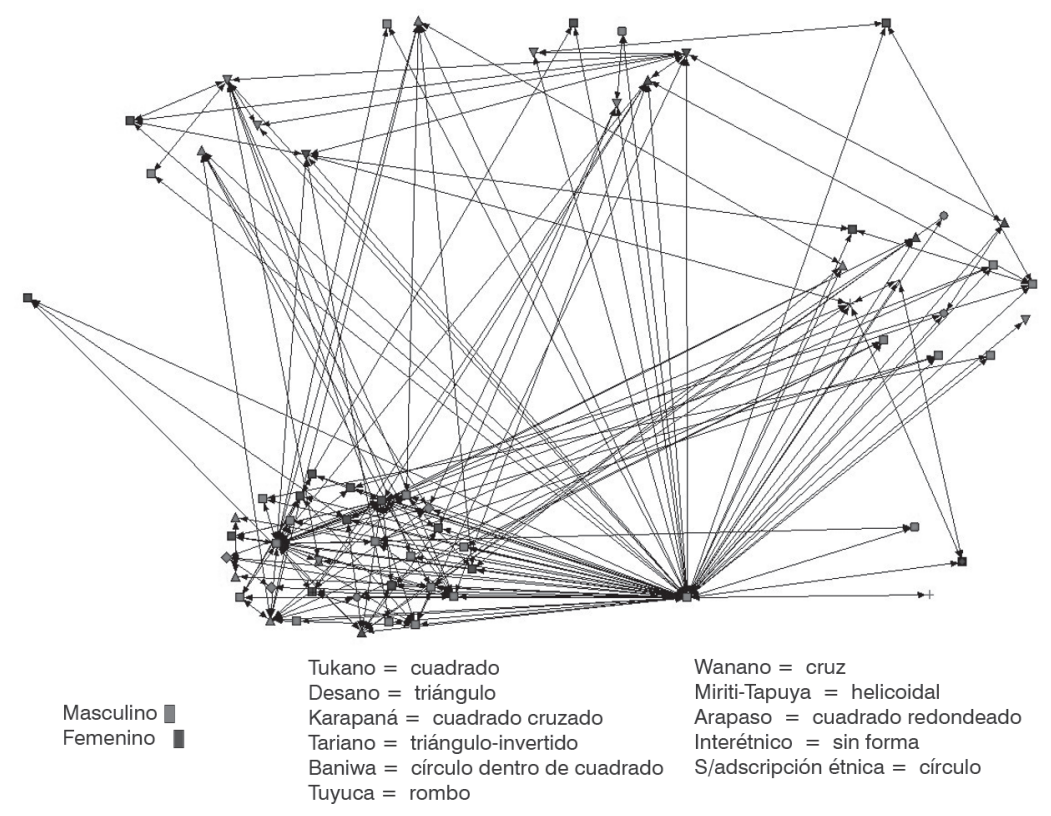


En cuanto a los vínculos por categoría étnica, verificamos que el mismo actor mantiene lazos con individuos que pertenecen a todas las etnias existentes en la ciudad de Manaus, procedentes de todas las sub-regiones de origen de los inmigrantes. El grafo presenta una "fragmentación étnica" por las regiones de origen (ver los diferentes formatos de las figuras de cada grupo), por ejemplo, el caso del Grupo 1 (São Gabriel), donde vemos muchos actores Tukanos (cuadrados) concentrados a la derecha, intercalados con Desanos (triángulos) y algunos Tuyuca (rombo). B.T. (10), de hecho, él mismo es Tukano (cuadrado), pero parece tener una red de contactos más diversa y amplia que sus compañeros de origen y etnia (con excepción de H.H. (83), que también forma parte de la red egocentrada de B.T. y muestra un perfil de conectividad semejante). Finalmente, también puede verse que el actor B.T. posee una red de contactos diversificada, de acuerdo con el sexo de los otros actores, independientemente de la adscripción étnica o de la región de origen. De este modo, se puede concluir que la centralidad más elevada de B.T. está fuertemente asociada (no implicando causalidad) a los atributos de sexo, etnia y región.

Esta condición de B.T. (10) es única en toda la red, y también sobrepasa a H.H. (83), el segundo actor más central. La red personal de H.H. (83) se compone de 48 nodos y no se distribuye de manera tan equilibrada como la de B.T. (10), pero cuenta en su red personal con agentes centrales fuertemente conectados, alcanzando potencialmente la casi totalidad de la red, posición que justifica el hecho de haber desempeñado papel destacado como informante clave en la investigación (Fig.3).

Aunque la red de H.H. (83) sea menos diversificada que la de B.T.(10), aún así desempeña un importante papel de intermediación y conectividad entre las diversas categorías étnicas y los individuos provenientes de diferentes localidades. En el diagrama superior podemos ver una diferente disposición de los agrupamientos

FIGURA 3

Red egocentrada de H.H. (83) según sexo, etnia y región

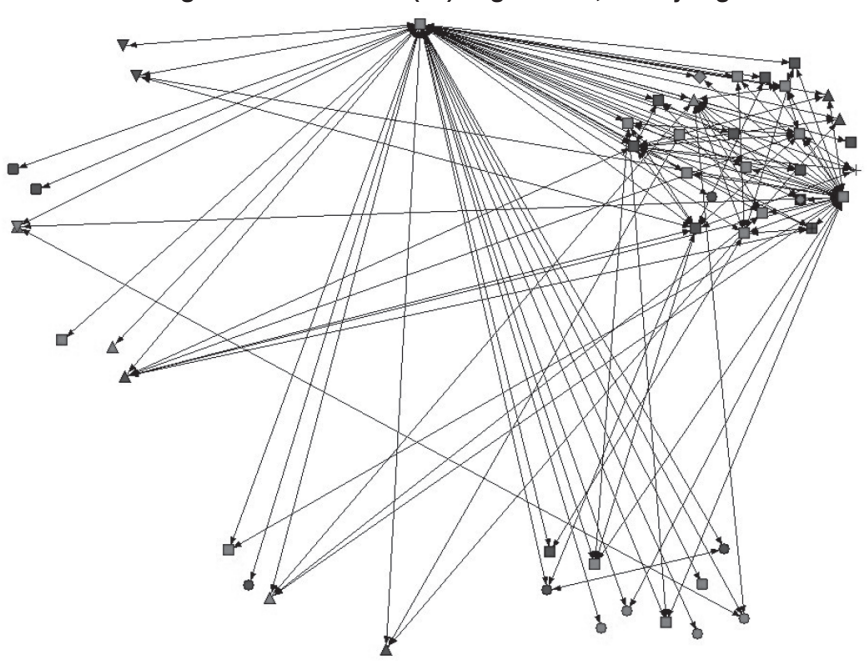

Tukano = cuadrado

Desano $=$ triángulo

Karapaná = cuadrado cruzado

Masculino

Tariano = triángulo-invertido

Wanano $=$ cruz

Femenino

Baniwa = círculo dentro de cuadrado

Tuyuca $=$ rombo

Miriti-Tapuya $=$ helicoida

Arapaso $=$ cuadrado redondeado

Interétnico $=\sin$ forma

S/adscripción étnica $=$ círculo

$$
\text { Tuyca = rombo }
$$


y la ausencia de un agrupamiento menor (procedente de Cucuí) en la red egocentrada [el orden de los agrupamientos en ese sociograma es el siguiente: el actor central en la parte más alta del grafo (H.H.), en el sentido horario, el Grupo 1 es procedente de São Gabriel; el Grupo 2 en la parte baja del grafo es de Parí-Cachoeira; el Grupo 3 de Maracajá-Sao Francisco; o Grupo 4 de Taraquá; el Grupo 5 de Yauareté; o Grupo 6 de Camarus; y el Grupo 7 sin identificación de procedencia].

La observación de las redes egocentradas de H.H. (83), según las cualidades de los lazos personales, muestra que sus contactos no son tan amplios y diversificados como B.T. (10). En especial, concentra más lazos con mujeres entre sus contactos personales cuando lo comparamos, por ejemplo, con las redes personales de otros actores. De hecho, uno de los motivos de la crisis establecida entre los indios y la congregación religiosa giraba en torno al gran número de mujeres solteras enviadas a los centros urbanos por los padres y monjas, según los indios, para realizar trabajos domésticos en residencias de autoridades y bienhechores de la orden. Efectivamente, H.H. (83) fue crucial en la identificación del grupo de mujeres jóvenes empleadas domésticas, asunto en el que ofreció subsidios estratégicos a B.T. (10) para la acción política de denuncia en el tribunal internacional.

En cuanto a los demás actores con alguna centralidad destacada, ninguno presenta inserción tan fuerte y amplia cuanto los actores B.T. (10) y H.H. (83). En los análisis hechos de índices y visualización, se percibe que los actores C.D. (34), F.T. (50), G.X. (73), K.M. (96) y M.N. (117) tienen roles preponderantes en regiones "localizadas" de la red. Son más o menos centrales, más o menos intermediarios, ellos poseen todos los recursos básicos para conectarse rápida y directamente con otros agentes muy bien relacionados en la red total (especialmente los agentes G.X. (73), M.N. (117) y C.D. (34).

\section{Análisis de la red según los atributos}

Finalmente, cuando analizamos las redes, total y egocentradas (de los agentes más centrales), podemos observar que la distribución de los arcos tiende a concentrarse sobre las categorías étnicas y también, principalmente, sobre la región de origen, evidenciando la persistencia de las lealtades étnicas y de regiones de origen (centros misioneros). A excepción de la red egocentrada de H.H. (83), que excepcionalmente reúne gran numero de contactos con agentes femeninos, el resto de los nodos analizados (y también cuando consideramos la red total) revelan gran concentración asociada a las cualidades étnicas y regionales.

La red estaba compuesta de individuos residentes en Manaus, originarios de los siguientes lugares del Alto Río Negro: la mayor parte (84\%) provenía de los ríos Tiquié (Misión Parí-Cachoeira) y Uaupés (Misiones Yauareté y Taraquá), un grupo de migrantes (16\%) había partido de los ríos Içana y Papurí, o desde otros núcleos urbanos próximos al área indígena como São Gabriel da Cachoeira, o ciudades intermediarias como Barcelos o Santa Isabel.

Junto a la diversidad de los puntos de partida de los migrantes, registramos una correspondiente variedad de identificaciones o adscripciones a categorías étnicas (intertribales), produciendo la siguiente composición:

En el contexto local urbano los individuos componen, no obstante, la diversidad de origen y étnica apuntada, un conjunto relativamente compacto, ejemplarmente expresado, por la extensa red de relaciones

Categorías étnicas - porcentaje

\begin{tabular}{|llll|ll|ll|}
\hline Tukano & 38,0 & Tuyuka & 3,0 & Pirá-tapuya & 2,0 & Baraçana & 1,0 \\
Tariano & 12,0 & Arapaso & 3,0 & Mirití-tapuya & 2,0 & Karapaná & 1,0 \\
Desano & 8,0 & Wanano & 3,0 & Baniwa & 1,0 & Sin adscrip. & 26,0 \\
\hline
\end{tabular}


que supone el mutuo conocimiento y el reconocimiento a la pertenencia de un universo social común. Podemos decir, con excepción de muy pocos casos, que todos los agentes se encuentran inscritos en una malla tribal urbana, unidos entre sí por una multiplicidad de enlaces: vínculos estrechados por lazos de parentesco generalmente, pero también amistad o cooperación establecida en algún momento de la migración, entre individuos originarios de diversos lugares y entre migrantes más "antiguos" y los más "nuevos". Veíamos reproducirse, en el medio urbano, con las modificaciones producidas por el propio desplazamiento del contingente, los lazos existentes entre esas unidades sociales, componiendo en la ciudad un todo social sin pérdida de las identidades específicas que operan en el área de origen. En ese sentido, la situación urbana nos propició un momento privilegiado para la observación de los procesos de identificación, tanto intertribal, como interétnico (indios y blancos), por constituir una nueva instancia de intensificación y reorganización de relaciones entre los grupos, muchos de los cuales geográficamente separados en el área indígena.

¿Cómo explicar la persistencia de esa multiplicidad de identidades étnicas categoriales cuando componen, al mismo tiempo, un articulado conjunto social y cultural ${ }^{9}$ Parece plausible comprender aquella multiplicidad de identificaciones diferentes y diferentemente asumidas por los "grupos discretos" como categorías étnicas que se definen mutuamente en el ámbito de relaciones intergrupales. Podemos afirmar, en ese sentido, que las identidades dichas "tribales" del Río Negro se presentan como posiciones en un sistema referencial de carácter ideológico, marcadas por relaciones de complementariedad, susceptibles de ser concebidas por los agentes como categorías étnicas en el límite del sistema intertribal.

\section{Trayecto espacial y trayectoria social}

Así, la migración indígena es, al mismo tiempo, resultante y locus del conflicto entre indios y no-indios. Por una parte, se presenta para los agentes como alternativa liberadora de la situación de dominación vivida en el área de origen; por otra, reproduce, de modo diferente pero con el mismo vigor, las relaciones de confrontación entre las dos sociedades. ${ }^{10}$

El trayecto espacial recorrido por la migración es al mismo tiempo generador de una trayectoria social. El desplazamiento espacial es responsable de una percepción común sobre las causas y características del propio movimiento poblacional y de las relaciones entre las etnias que toman lugar en ese proceso. De hecho, podemos tomar la migración como un fenómeno colectivo con un efecto particular para los actores, el de reforzar la trayectoria social común de los diversos grupos indígenas que se desplazan mediante una efectiva comunión de condiciones enfrentadas a lo largo de todo el proceso migratorio. La trayectoria común también contribuye a una representación de los conflictos mantenidos con la sociedad nacional -que no es otra cosa que una representación de una posición común objetivamente ocupada en la estructuración social como un todo.

El proceso migratorio que analizamos en los puntos precedentes posibilitó captar lo que tiene de específico del movimiento de población indígena emigrada del Alto Río Negro, permitiendo aprehender las condiciones objetivamente compartidas por los agentes. Por esa razón y por reproducir

\footnotetext{
${ }^{9}$ Hemos visto que el criterio lingüístico utilizado como co-término de tribu o cultura no se aplica para los grupos del Río Negro. La lengua Tukano es extensamente usada como lengua franca, por otro lado, existen grupos que han perdido su identidad lingüística (los Tariano) y hoy hablan solamente Tukano, finalmente el caso Kobewa pone en tela de juicio la correlación lengua y grupo exogámico y, con eso, los límites tribales.

${ }_{10}$ Acerca de este punto A. Sayad (1998) sugiere que la migración, como un hecho social total, representa la incorporación por el migrante de la lógica práctica colectiva, que responde a los conflictos externos y sostiene las jerarquías y relaciones internas. El proyecto migratorio, según Sayad, representa las contradicciones y paradojas de las relaciones asimétricas entre grupos sociales y sus agentes. En efecto, los desplazamientos son resultado de los conflictos en la colectividad al mismo tiempo que son el refuerzo de tales conflictos en las trayectorias sociales de los agentes.
} 
en su interior relaciones de conflicto entre indios y no-indios, la migración constituye una instancia social de convergencia identificatoria. O sea, si el desplazamiento hacia la ciudad genera un tipo de desunión de los agentes con sus comunidades de origen, se comporta también como tendencia aglutinadora que lleva a una fuerte identificación, pues la homogeneidad migratoria saca a la luz la idéntica posición de los agentes en la estructura social -lo cual resulta no solamente de una comunión de relaciones objetivas mantenidas con la sociedad dominante, sino también de una identidad de relaciones vividas que se experimentan con esas condiciones objetivas.

La regularidad social objetiva que constituye la práctica migratoria, regularidad que aprehendemos estadísticamente en el curso del análisis de las varias fases del proceso migratorio, da lugar a una representación que envuelve esa práctica, representación que por su carácter identificador actúa como mecanismo integrador, propiciando recursos prácticos y simbólicos suficientemente eficaces a punto de compensar, en el plano interno da sociedad indígena, los desequilibrios que surgen como resultados de los contactos e intercambios que se efectúan entre agentes situados en posiciones sociales asimétricas. En ese sentido, se puede afirmar que la identidad étnica se completa, o rehace, en el interior del proceso migratorio, pues se reproducen allí formas da dominación impuestas a la sociedad tribal; se trata de la construcción de una identidad configurada bajo símbolos étnicos que reposa en una práctica común, identidad que sirve para expresar-como dice Bourdieu- ciertas demandas de significado y constituye tal expresión en la medida que ofrece a sus portadores los materiales significantes con los cuales se vehiculan las significaciones orientadas por los intereses y reivindicaciones de ese grupo (1974: LXI).

Se delimitan, así, los trazos pertinentes que, por contraste, recortan y hacen aparecer los límites del grupo, trazos que van tomando su forma a lo largo de la historia del contacto y se patentizan durante la migración. La construcción social de esos límites, colectivamente elaborados en el interior del juego dialéctico entre indios y blancos tiene por efecto, para la sociedad indígena, provocar la disminución de las diferencias entre las categorías étnicas -componentes de la corriente migrante saliente del Río Negro- y todo ello materializado en la compleja y muy conectada red urbana, llevando a la adopción de una visión común, socialmente definida, de la división étnica del mundo social marcado por el conflicto con la sociedad dominante.

Todo ocurre como si los agentes pasasen de una previa experiencia de comunidad a una comunidad de experiencia, pasaje que les permite descubrir las propiedades comunes vis-a-vis la sociedad nacional, por encima de la diversidad de las situaciones particulares que aíslan, dividen o desmovilizan, y construir su identidad social sobre la base de trazos o experiencias que se constituyen en francos índices de pertenencia al mismo grupo.

Construido antagónicamente, el grupo se redefine sobre las bases de las experiencias comunes aprehendidas en la situación crítica generada por el movimiento migratorio y por la distancia social que los separa, colectivamente, de la sociedad dominante. Se puede decir que la migración es, en sí misma, la expresión espacial que toma el conflicto entre la sociedad indígena y la sociedad nacional, contribuyendo, en última instancia, a la disminución o suspensión de las diferencias intertribales, desorganizadas por el contacto, auxiliando en la reconstrucción de los límites grupales pues, como instancia social práctica, constituye una matriz que refuerza la solidariedad del grupo vivida durante la migración, definida en contradicción al orden global dominante. La trayectoria espacial migratoria es, vista de esta manera, un encadenamiento físico profundamente marcado por una oposición étnica que resulta de una ideación colectiva de experiencias socialmente acumuladas, la cual hace del espacio geográfico un espacio social étnicamente dividido. La red urbana tribal, que incorpora a los lazos constantes del parentesco y de las lealtades étnicas aquellos trabados durante el desplazamiento migratorio, no es más que la impronta, la 
huella, de esa trayectoria común generadora de una nueva percepción de las relaciones étnicas.

Siendo así, el fenómeno migratorio indígena constituye un momento social crítico por el que se redimensionan, por la redimensión física que toda migración supone, las relaciones sociales y las correspondientes identificaciones, tanto entre los grupos tribales que componen la totalidad del flujo que se desplaza hacia la ciudad, como de estos con la sociedad nacional. El contacto interétnico, entre indios y blancos, afectó el sistema de categorías étnicas y sus respectivas identificaciones. Esta situación hizo posible la emergencia de una identidad étnica inclusiva, la de Indios del Alto Río Negro que encontrábamos al comienzo de la investigación, cristalizada en la red de relaciones, alimentada por los migrantes y construida sobre los ejes de un espacio y una historia no-indígena, arbitrariamente impuestos por la sociedad nacional.

Al abordar, por una parte, el complejo de categorías étnicas de los Indios del Alto Río Negro por medio del registro de la malla de lazos sociales, de un grupo de migrantes muy dispersos en el área urbana, $\mathrm{y}$, por otra parte, siendo ese registro más que una técnica de investigación, la propia evidencia de la continuidad de las identidades étnicas específicas, en condiciones de "destribalización", articuladas además a una

\section{References}

AGUILAR, C.; MOLINA, J. L. Identidad étnica y redes personales entre jóvenes de Sarajevo. REDES, v. 7, \#6, Oct./Nov. 2004 $<$ http://revista-redes.rediris.es $>$.

BARNES, J.A. Networks and political process. In: MITCHELL, J.C. (Ed.). Social networks in urban setting.. Manchester, England: Manchester University Press, 1954, p. 51-76.

BOURDIEU, P. A economia das trocas simbólicas. São Paulo: Perspectiva, 1974.

BOTT, E. Family and social network. Londres: Tavistock, 1957.

BRANDHUBER, G. Why Tukanoans migrate? Some remarks on conflict on the Upper Rio identidad inclusiva, generada en el contexto del desplazamiento migratorio, atendíamos lo que considerábamos la propiedad característica de las identidades sociales: su carácter contrastivo y, sobre todo, relacional (Cardoso de Oliveira, 1976).

Creemos, con Edmund Leach, que cuando diferenciamos los miembros de una población humana, o sea, apuntamos discontinuidades sociales de algún tipo, en términos de algún parámetro como los morfológicos, lenguaje, costumbres, religión, etc., son siempre objeto de una definición escogida que de hechos evidentes. Siempre que nos servimos de tales discriminaciones, advierte el autor, para ordenar el universo social, los límites que de ahí resultan son el producto de un evidente prejuicio cultural (1985). La estrategia escogida de trazar las redes sociales para el estudio de ese complejo universo indígena, se mostró una herramienta preciosa para neutralizar desvíos de ese tipo y el error más frecuente en los estudios de grupos étnicos, producto de perspectivas esencialistas (Aguilar, C. \& J. L. Molina, 2004), permitiéndonos aprehender el fenómeno del desplazamiento migratorio, la persistencia de las fronteras étnicas intertribales y la articulación con nuevas formas de percepción e identificación frente a la sociedad nacional, desde la mirada, que junto a Emirbayer (1997), podemos denominar una genuina antropología relacional.

Negro (Brazil). Jornal de la Societé des Américanistes, 85, p.261:280, 1999.

CARDOSO DE OLIVEIRA, R. Urbanização e tribalismo: a integración dos índios Terêna numa sociedade de classes. Rio de Janeiro: Zahar, 1968.

O índio e o mundo dos brancos.

São Paulo: Biblioteca Pioneira de Ciências Sociais, 1972.

Identidade, etnia e estrutura social. São Paulo: Biblioteca Pioneira de Ciências Sociais, 1976.

CASTELLS, M. Problemas de investigación en sociología urbana. Buenos Aires: Siglo XXI, 1972. 
EISENSTAD, S. N. The absortion of inmigrants: a comparative study bases mainly on the jewish comunity in Palestine and the State of the Israel. Londres: Routledge \& Kegan Paul, 1954.

EMIRBAYER, M. Manifesto for a relational sociology. American of Journal of Sociology, 103, n. 2 (September): 281317, 1997.

FAZITO, D. Reflexões sobre os sistemas de migração internacional: proposta para uma análise estrutural dos mecanismos intermediários. Tese (Doutorado). Belo Horizonte, Cedeplar, Universidade Federal de Minas Gerais (UFMG) 2005.

FíGOLI, L.H.G. Identidad étnica y regional: trayecto constitutivo de una identidad social.. Dissertação (Mestrado). Brasilia, Universidade de Brasília (UnB), 1982.

GALVÃO, E. Encontro de sociedades. Rio de Janeiro: Paz e Terra, 1979.

GOLDMAN, I. The Cubeos: indians of the northwest amazon. Illinois Studies in Anthropology, 2, Illinois: University of Illinois Press, 1963.

HUGH-JONES, S. The palm and the pleiades. Initiation and Cosmology in Northwest Amazonia. Great Britain: Cambridge University Press, 1979.

JACKSON .J. Language identity of the colombian vaupés indians. In: RICHARD, B.; SHERZES, J. (Eds.). Explorations in the ethnography of speaking. USA: Cambridge University Press, 1979.

KILLWORTH, P.; JONHSEN, E.; MCCARTY, C.; ASHLEY, G.A.; BERNARD, H.R. A social network approach to estimating seroprevalence in the United Status. Social Networks, 20:23-50, 1998.

KNOKE, D.; KUKLINSKY, J. Network analysis. London SAGE, 1983.
LEACH, E. Anthropos. Enciclopedia Einaudi. Lisboa: Imprensa Nacional-Casa da Moeda, v. V, 1985.

McCARTY, C. Structure in personal networks. Journal of Social Structure, 2002.

McCARTY, C. et al. Eliciting representative samples of personal networks. Social Networks, v.19, p. 303-23, 1997.

MITCHELL, J.C.Social networks in urban setting. Manchester, England: Manchester University Press, 1969.

MITCHELL, J. C. Orientaciones teóricas de los estudios urbanos en África. In: WOLF et al. Antropología social de las sociedades complejas. Madrid: Alianza, 1980.

SCOTT, J. Social network analysis, a handbook. London: SAGE, 2000.

WASSERMAN, S.; FAUST, K. Social network analysis: methods and applications. Cambridge: Cambridge University, 1994.

WELLMAN, B. Structural analysis: from method and metaphor to theory and substance. In: SCOTT, J. (Ed.). Social networks: critical concepts in sociology. New York: Routledge. v.1, 2002, p.81-122.

WOLF et al. Antropología social de las sociedades complejas. Madrid: Alianza, 1980.

REICHEL-DOLMATOFF, G. Amazonian cosmos: the sexual and religious symbolism of the Tukano indians. Chicago: Chicago Press, 1973.

SAYAD, A. A imigração ou os paradoxos da alteridade. São Paulo, Edusp: 1998.

SORENSEN, A. A Multilingualism in the Northwest Amazon. American Anthropologist, 69(6), 1967.

\section{Resumo}

Redes sociais em uma investigação de migração indígena: o caso de Manaus

Este trabalho pretende discutir alguns aspectos metodológicos do mapeamento de redes sociais com migrantes indígenas, a partir de uma pesquisa etnográfica realizada junto a um grupo de 
migrantes índios (Alto Rio Negro) e moradores de Manaus (Amazônia). O desenho das redes sociais constituiu um instrumento de campo, permitindo identificar os indivíduos dispersos no local de destino da migração, obter dados sobre os modos de deslocamento ou de instalação dos migrantes, na área urbana. Além de, por um lado, mostrar-se importante instrumento de pesquisa, também revelou-se um conceito fundamental, do ponto de vista teórico, pois as próprias redes desenhadas constituíam forte evidência de coesão social, manutenção e reelaboração dos laços étnicos, no contexto urbano. As redes mapeadas, por exemplo, revelaram a maleabilidade das fronteiras étnicas desenhadas pelos grupos, resultado das relações sociais desenvolvidas durante o percurso migratório, o que nos obrigou a apreender o fenômeno étnico desde a perspectiva de uma genuína antropologia relacional. Hoje, as novas tecnologias informatizadas permitem melhor visualização e análise mais detalhada das redes sociais (ARS), do que foi possível realizar manualmente, na época da pesquisa (1980). Os novos recursos tornam ainda mais relevante essa ferramenta na pesquisa etnográfica. A proposta deste trabalho é discutir suas possibilidades e aplicações no estudo de fluxos migratórios indígenas.

Palavras-chave: Migração indígena. Redes sociais. Amazônia. Identidade étnica. Urbanização.

\section{Abstract}

Social networks in a study on migration of Brazilian Indians: the case of the city of Manaus

This article discusses several methodological aspects of the work of mapping social networks of migrant Brazilian Indians, based on an ethnographic study carried out on a group of migrant Indians (from the Upper Rio Negro, in Amazonian Brazil) and dwellers in the city of Manaus, also in the Amazon). The characteristics of the social networks of this population constituted an instrument for field study, making it possible to identify the individuals scattered throughout their destination area and obtain data on their systems of displacement and settlement in the urban area. This tool proved, on the one hand, to be an important research instrument and, on the other, a basic concept from the theoretical point of view. The networks themselves were seen to have strong social cohesion as they dealt with the maintenance and reorganization of their ethnic ties in an urban context. For example, the networks we charted indicated the malleability of ethnic boundaries established by the groups as the result of social relationships developed during the migration period. This obliged the researchers to apprehend the ethnic phenomena involved from the perspective of a true relational anthropology. Today new computerized technologies allow better visualization and more detailed analysis of social networks than was possible at the time of the study, in 1980. The new resources prove that this tool is even more significant in ethnographic research. The objective of the article is to discuss the tool's possibilities and applications in the study of migratory flows of Brazilian Indians.

Keywords: Indigenous migration. Social networks. Amazon. Ethnic identity. Urbanization. 